\title{
Proyecto "Restauración de vestigios de artillería": aspectos sobre la investigación y conservación de cañones con aleación de hierro de la ciudad de San Francisco de Campeche
}

\author{
Diana Arano Recio
}

a ciudad de San Francisco de Campeche, México, demuestra su magnificencia con los ejemplos de arquitectura civil, militar y religiosa que ornamentan su centro histórico y los barrios antiguos, y por medio de innumerables bienes muebles como los archivos parroquiales y civiles, obras de arte sacro y la artillería que se exhibe en los museos y calles de la ciudad. De ahí que la Organización de las Naciones Unidas para la Educación, la Ciencia y la Cultura (UNESCO) la declarara en 1999 Patrimonio Mundial de la Humanidad.

En la Sección de Restauración del Centro INAH (Instituto Nacional de Antropología e Historia) Campeche, a sus casi 10 años de creación, hemos observado que, a causa de la posición geográfica de la ciudad y del tipo de clima imperante en la región, la velocidad de los mecanismos de degradación de los materiales que constituyen al patrimonio cultural es mucho mayor que en los lugares con clima templado, lo que ha orillado a buscar fuera del instituto asesoría en materia de ciencia aplicada a la preservación y conservación, así como a crear programas de mantenimiento de colecciones, como es el caso de la de cañones expuestos en lugares públicos de la capital del estado.

\section{Antecedentes}

En los barrios antiguos de la ciudad de Campeche, en glorietas, en algunas esquinas, en baluartes, en los fuertes y en el malecón es posible observar la colección de 107 cañones coloniales inventariados hasta el momento por la sede regional de Arqueología Subacuática del INAH, a la que se suman dos donaciones, recibidas por la Dirección de Museos del Centro INAH-Campeche, de objetos hallados durante las actividades cotidianas del puerto y la construcción de edificaciones modernas: dos cañones y un ancla, hecha por pescadores, y un cañón más, que la Comisión Nacional de Agua Potable encontró durante el desarrollo de obras (Carvajal 2004). 


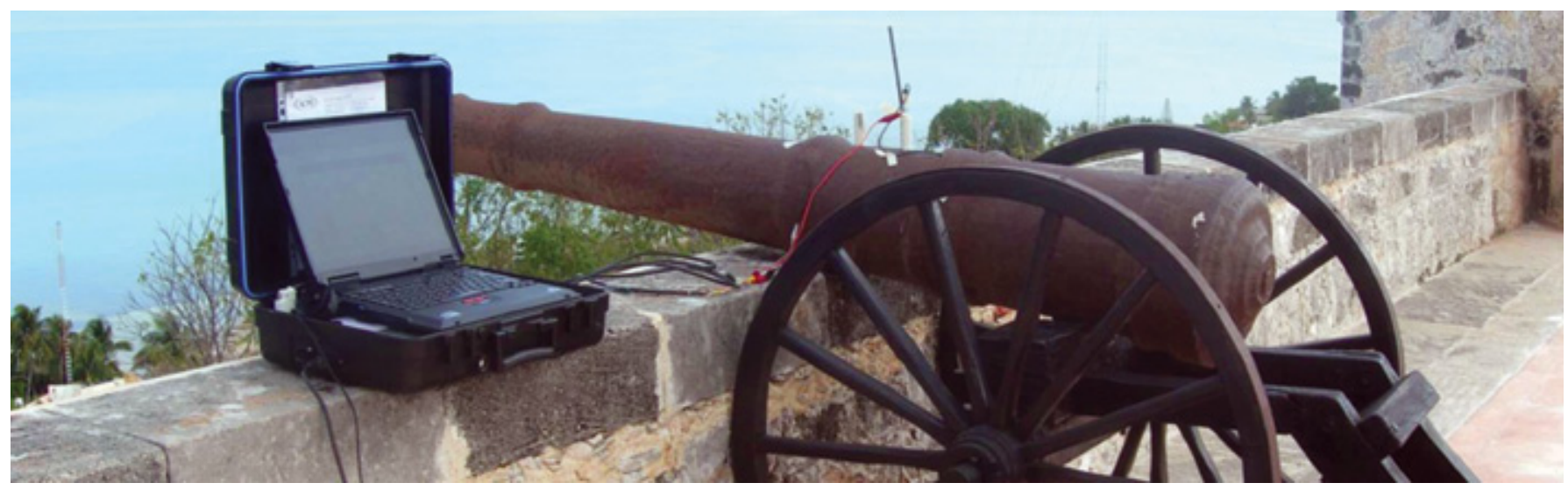

FIGURA 1. Realización de pruebas de polarización potenciodinámica in situ en uno de los cañones del Fuerte de San Miguel durante la etapa experimental del proyecto del Cicorr (2005). El potenciostato de campo Field Machine está conectado al cañón como electrodo de trabajo, al electrodo de referencia AG $\mid \mathrm{AgCl}$ y al auxiliar; como electrolito se utilizó agua Iluvia (Fuente: Arano Recio 2008; cortesía: Archivo Fotográfico de la Sección de Restauración del Centro INAH-Campeche).

Desde 2005, cuando se estableció en esa entidad un convenio entre la delegación estatal del INAH y la Universidad Autónoma de Campeche (UAC), específicamente, con su Centro de Investigaciones en Corrosión (Cicorr), se han llevado a cabo varios proyectos de investigación en pro de la conservación del patrimonio cultural. Uno de ellos: "Determinación del grado de deterioro y aplicación de métodos de preservación de piezas metálicas consideradas como patrimonio cultural de la ciudad de Campeche", que contó con financiamiento externo a través del Fondo Mixto de Fomento a la Investigación Científica y Tecnológica ConacytGobierno del Estado de Campeche, convocatoria 2005 (González Sánchez 2005), establece varias finalidades: crear un catálogo de bienes culturales manufacturados en metal, identificar y evaluar el estado de conservación del material que constituye a estos bienes, y establecer e instrumentar tratamientos viables para su conservación (González Sánchez 2005).

Dentro de este proyecto, la que suscribe desarrolló, para obtener el título de maestra en Ciencias Marinas, la investigación "Conservación de cañones de fundición de hierro del periodo colonial que forman parte del patrimonio cultural de la ciudad de San Francisco de Campe- che", que abarca estudios sobre la microestructura, la identificación de aleación, la caracterización de productos de corrosión, la determinación de la agresividad de la ciudad de Campeche, la implementación de un tratamiento químico-electroquímico de formación o estabilización de productos de corrosión en cañones y la evaluación de la resistencia a la corrosión de piezas metálicas con tratamiento de formación/estabilización de productos de corrosión (Arano Recio 2008). De este estudio, los resultados de las pruebas de polarización potenciodinámica (Arano 2008) ${ }^{1}$ demuestran que el tratamiento de estabilización, que consiste principalmente en la eliminación de productos de corrosión, la formación de una capa delgada y homogénea de corrosión con una solución de ácido nítrico $1 \mathrm{~mol}$ y la estabilización de esa capa con una solución de ácido tánico al 5\% en alcohol metílico, reduce hasta 10 veces la velocidad de corrosión en los cañones que no pre-

${ }^{1}$ La polarización potenciodinámica es una técnica de análisis electroquímico de corriente directa, donde se imprime corriente al electrodo de trabajo para obtener una variación en su potencial de corrosión. Como resultado se obtiene un gráfico, conocido como curva de polarización, que cifra la variación del potencial de corrosión respecto de la densidad de corriente. sentan contaminación por cloruros (Figura 1).

\section{Los cañones de la ciudad de San Francisco de Campeche}

Durante buena parte del siglo $\mathrm{xx}$ el INAH, por medio de su delegación estatal en Campeche, distribuyó y conservó varios cañones en edificaciones bajo su jurisdicción, mientras que el ayuntamiento de la ciudad colocó algunos, como un emblema de su historia, en los principales barrios antiguos, ya para delimitarlos, ya -en algunos casos, no muy afortunados-para proteger a las casas de golpes de automóviles en calles muy estrechas, en cuyas esquinas se sembraron verticalmente. Otros ejemplares han quedado en manos de la Armada y del Ejército mexicanos, en tanto que otros más se encuentran en colecciones particulares.

Casi la totalidad de los cañones y piezas metálicas registrados $(96 \%)$ está en exhibición al aire libre, en su mayoría a una distancia menor a 3 $\mathrm{km}$ de la línea costera y en otros casos, como los del Baluarte de San Francisco, cerca de uno de los cruces viales más transitados de la ciudad.

Estos ejemplos de la artillería colonial, admirados por los habitantes de la ciudad y por los visitantes nacionales y extranjeros, han quedado inmersos en la vida cotidiana del 
puerto como parte importante del legado cultural de los campechanos al mundo.

Identificación de los materiales constitutivos y la técnica de manufactura

La época colonial, de la que data la artillería en la península de Yucatán, heredó a los siglos posteriores su tecnología y desarrollo: se cree que la mayoría de las piezas que se conservan actualmente en la ciudad de Campeche se fabricó en España, si bien es posible que, por los ataques de corsarios a este puerto, algunas de ellas provengan de Inglaterra y Francia (Díaz Fuentes 2008).

En el siglo XVI casi todas estas armas se fabricaban en hierro, y no fue sino hasta el siglo XVIII cuando se consiguieron mejoras importantes en la metalurgia de este elemento: una de las innovaciones fue la obtención de cañones de segunda fusión, esto es, piezas logradas de la refundición en hornos de reverbero ${ }^{2}$ de distintas clases de hierro de primera fusión, operación por medio de la cual se eliminó gran parte de los cuerpos simples que perjudican la resistencia del hierro colado. Se mejoró asimismo la manera de conformar la aleación hierro-carbono, mediante cambios de temperatura de los hornos y durante el proceso de enfriamiento (Carrero Blanco s. f.).

De acuerdo con los resultados de la investigación citada (Arano Recio 2008), la cual estudió la microestructura de cañones en exhibición en el Fuerte de San Miguel, se ha identificado que la aleación de los cañones corresponde a hierro colado gris; que su composición reside entre los intervalos $2.5-4 \%$ de carbono, $1-3 \%$ de silicio, $0.2-1 \%$ de manganeso, $0.02-$ $0.25 \%$ de azufre y $0.02-1 \%$ de fósforo, cuya morfología es de grafito de los tipos A, B y C (Kumar 2003) sin

\footnotetext{
${ }^{2}$ Horno rectangular cubierto por una bóveda de ladrillo refractario; el calor producido por la combustión escapaba por medio de una chimenea.
}

ningún tratamiento térmico. La microestructura de los cañones consta de una matriz continua de ferrita con colonias de perlita, y en algunos se presenta la steadita, que es una fase eutéctica con fósforo binario. Cada una de las fases que constituyen la microestructura del hierro colado gris posee una diferencia mínima en potencial de corrosión, cualidad que da lugar a que la destrucción de los cuerpos metálicos en este tipo de aleación sea casi instantánea, al reaccionar con una capa microscópica de humedad.

\section{Condiciones climáticas y agresividad atmosférica de la ciudad de Campeche}

La corrosión atmosférica no depende solamente de la temperatura, cuyo promedio anual en la ciudad de Campeche es de $27^{\circ} \mathrm{C}$, y la máxima histórica de $47^{\circ} \mathrm{C}$ (Inegi 2008), sino también de la presencia de humedad (la temporada de lluvias está comprendida entre los meses de junio y octubre, y la de estiaje de enero a mediados de mayo). El incremento de la temperatura ambiental disminuye la posibilidad de que exista condensación de agua en las piezas metálicas, debido a que con la radiación solar el metal se calienta y la humedad en superficie se evapora. Para que se dé el fenómeno de condensación en una superficie metálica es necesario que la humedad relativa (HR) esté por encima de $80 \%$-porcentaje promedio anual en esta ciudad, que registra una precipitación pluvial anual de $1119.6 \mathrm{~mm}$ (Inegi 2008) - y entre $40 \%$ y $50 \%$ en presencia de cloruros (Veleva 2003).

Estos datos son esenciales, en tanto que la duración del proceso de corrosión depende del lapso que permanezca sobre la superficie del metal una capa de humedad (electrolito), cuyo espesor varía desde zonas muy delgadas (invisibles) hasta aquellas que mojan perceptiblemente el metal (Reyes 1999).

Aunado a lo anterior, como Campeche es una ciudad costera, los cañones que están en exhibición a la intemperie se exponen a los aerosoles marinos ${ }^{3} y$, a la brisa, los que están en el malecón.

En los trópicos existen elevados intervalos de temperatura y de humedad, una intensa radiación solar y largos periodos de condensación durante las noches. Si bien la atmósfera de Campeche, comparada con la de otras ciudades del Golfo de México, puede ser ligeramente menos corrosiva, debido a que el aporte de cloruros es menor por la dirección de los vientos predominantes, es considerada, según las normas ISO (ISO/ WD 9223), una atmósfera con agresividad media alta.

\section{Evaluación de los bienes culturales}

El estado de conservación de los cañones depende de varios factores: su procedencia y la posición en que se encontraban en su contexto original, ya fuese marino o terrestre; su ubicación actual en el sitio de exhibición (cercanía o lejanía de la costa marina, presencia de tránsito vehicular y tiempo de incidencia de luz solar), así como el tiempo que han permanecido en ésta y los tratamientos de estabilización a los que se han sometido.

Por su microestructura, los objetos de hierro colado, como es el caso de los cañones manufacturados durante el periodo colonial, tienden a su estado natural, para lo cual se combinan con otros elementos en la generación de óxidos e hidróxidos de hierro que forman una gruesa capa, con menor densidad y mayor volumen que la que originalmente presentaba el hierro metálico. En el caso de los cañones del Fuerte de San Miguel se encontró la presencia de otros iones en los productos de corrosión, como sílice $(\mathrm{Si})$, calcio $(\mathrm{Ca})$, cloro $(\mathrm{Cl})$, manganeso $(\mathrm{Mn}) \mathrm{y}$, en proporciones menores a $1 \%$ PV, magnesio $(\mathrm{Mg})$, azufre $(\mathrm{S})$, potasio $(\mathrm{K})$, sodio $(\mathrm{Na})$ y

\footnotetext{
${ }^{3}$ Los aerosoles son partículas de tamaño microscópico o submicroscópico (10-7 m) que se encuentran suspendidas en el aire.
} 
fósforo (P). En conjunto con el hierro y oxígeno dichos productos están conformando fases cristalinas, como cuarzo $\left(\mathrm{SiO}_{2}\right)$, goethita $(\mathrm{FeO}(\mathrm{OH}))$, la maguemita $\left(\mathrm{X}-\mathrm{Fe} 2 \mathrm{O}_{3}\right)$ y la akaganeita $(\beta-\mathrm{FeO}(\mathrm{OH}))$, principalmente (Arano Recio 2008).

Como era de esperarse, los cañones expuestos a las condiciones atmosféricas de la ciudad de San Francisco de Campeche presentan capas de productos de corrosión que en conjunto llegan a medir $3 \mathrm{~cm}$ de espesor, en el caso de los cañones clasificados en mal estado de conservación, y de $1 \mathrm{~cm}$ de grosor en el de los catalogados en estado regular. Estas capas están agrietadas, con desprendimientos localizados; algunas son más porosas y vulnerables al tacto que otras. Varios cañones evidencian sobre dichas capas una película de pintura comercial negra, ya desgastada en la parte superior por la incidencia de radiación solar.

En todos los casos las capas de productos de corrosión son permeables y receptoras de agua de precipitación pluvial, y ante la presencia de humedad las reacciones electroquímicas de corrosión continúan.

En los cañones con estado de conservación deplorable se infiere la presencia de compuestos de cloruro, que al ser higroscópicos absorben humedad ambiental incluso cuando los parámetros de HR son menores a 60\% (Veleva 2003), además de que se observa el goteo de un líquido de color anaranjado que escurre a través de las grietas en la capa de corrosión. Durante la primera etapa del proyecto "Restauración de vestigios de artillería" la medición del potencial de hidrógeno $(\mathrm{pH})$ en los cañones que presentaron escurrimientos dio como resultado una acidez de 2-4, con lo cual se comprueba la formación de un compuesto ácido (posiblemente, clorhídrico) que cataliza la reacción de corrosión.

\section{Programa de mantenimiento}

A lo largo del desarrollo de la investigación se evaluó, en colaboración con el Cicorr, un tratamiento de estabilización de herrumbre que, por primera ocasión, ofreció la oportunidad de cuantificar la disminución de la velocidad de corrosión una vez aplicado el tratamiento. Mediante las pruebas de polarización potenciodinámica se pudo apreciar cómo un cañón muestra, que observó un potencial de corrosión de $-356.32 \mathrm{mV}$ a los 36 días posteriores al tratamiento de estabilización, presentó un potencial de $18.33 \mathrm{mV}$, esto es, mucho más noble que sin el tratamiento. En términos de velocidad de corrosión, se degradaba $0.32 \mathrm{~mm} / a n ̃ o$, alcanzó después del procedimiento un rango de 0.0043 mm/año. el mismo cañón, que sin tratamiento

Una vez concluida de manera exitosa la investigación, nos enfrentamos a la necesidad inminente de brindar mantenimiento oportuno a una colección de 107 cañones manufacturados en hierro colado gris y distribuida en espacios públicos en diferentes puntos de la ciudad, lo cual representó un reto importante: el de aplicar una metodología evaluada a pequeña escala en el laboratorio a la restauración de un gran número de cañones en vía pública $y$ al aire libre (Figura 2). El personal de la Sección de Restauración del Centro INAH-Campeche no era suficiente para esta tarea, no obstante se llevó a cabo gracias a la colaboración de otras dependencias.

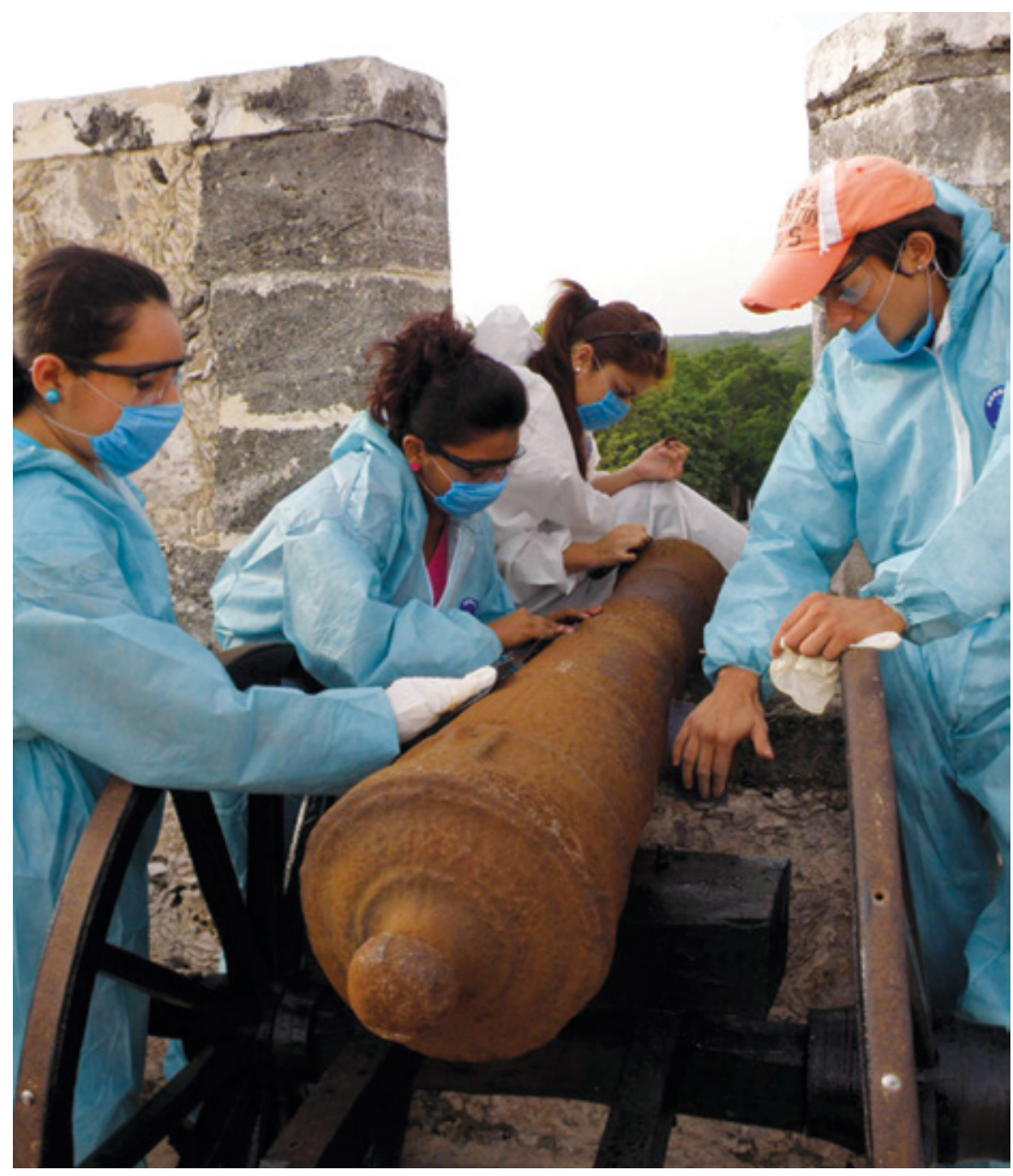

FIGURA 2. Eliminación de productos de corrosión en un cañón del Fuerte de San Miguel durante la primera etapa del proyecto "Restauración de vestigios de artillería" (Fuente: Arano Recio 2008; cortesía: Archivo Fotográfico de la Sección de Restauración del Centro INAH-Campeche). 
Proyecto "Restauración de vestigios de artillería"

Así fue como la Dirección y la Administración del Centro INAH-Campeche nos hicieron la propuesta de realizar un proyecto de "Restauración de vestigios de artillería", con el apoyo del programa de la Secretaría de Desarrollo Social (Sedesol) de Empleo Temporal, el cual ayuda económicamente a las personas que, afectadas por la baja oferta laboral o por fenómenos naturales, participan en proyectos de beneficio familiar o comunitario.

En el periodo de julio a octubre de 2009 se ejecutó la primera etapa del proyecto mencionado -durante la cual se seleccionaron los cañones para intervenir: tanto aquellos que están bajo la jurisdicción del Centro INAH local como los que se encuentran en mal estado de conservación, de acuerdo con el Catálogo de Bienes Culturales Metálicos de la Ciudad de San Francisco de Campeche, Campeche (Díaz Fuentes 2008)—, con recursos mixtos de la Sedesol federal y del proyecto específico del Centro INAH "Conservación de colecciones", dirigido por su Sección de Restauración. En esta primera temporada, con un equipo de 25 personas que recibieron la capacitación necesaria para realizar un trabajo especializado, se logró la conservación de 46 cañones, 41 balas de cañón, 1 ancla de almirantazgo y 1 caja fuerte, manufacturados en hierro durante el periodo colonial, que forman parte de las colecciones de los museos de Arqueología del Fuerte de San Miguel, de Barcos y Armas del Reducto de San José el Alto, de la Dirección de Museos del Centro INAH-Campeche, del Centro de Convenciones Campeche XXI y del Centro Cultural EI Polvorín, que se encuentran en exhibición en el Barrio de San Román y en el malecón de la ciudad (Figura 3).

Debido al éxito de la primera temporada del proyecto, el año siguiente se abrió una nueva que, con el trabajo de un equipo de 15 personas, restauró 37 cañones y 20 balas de

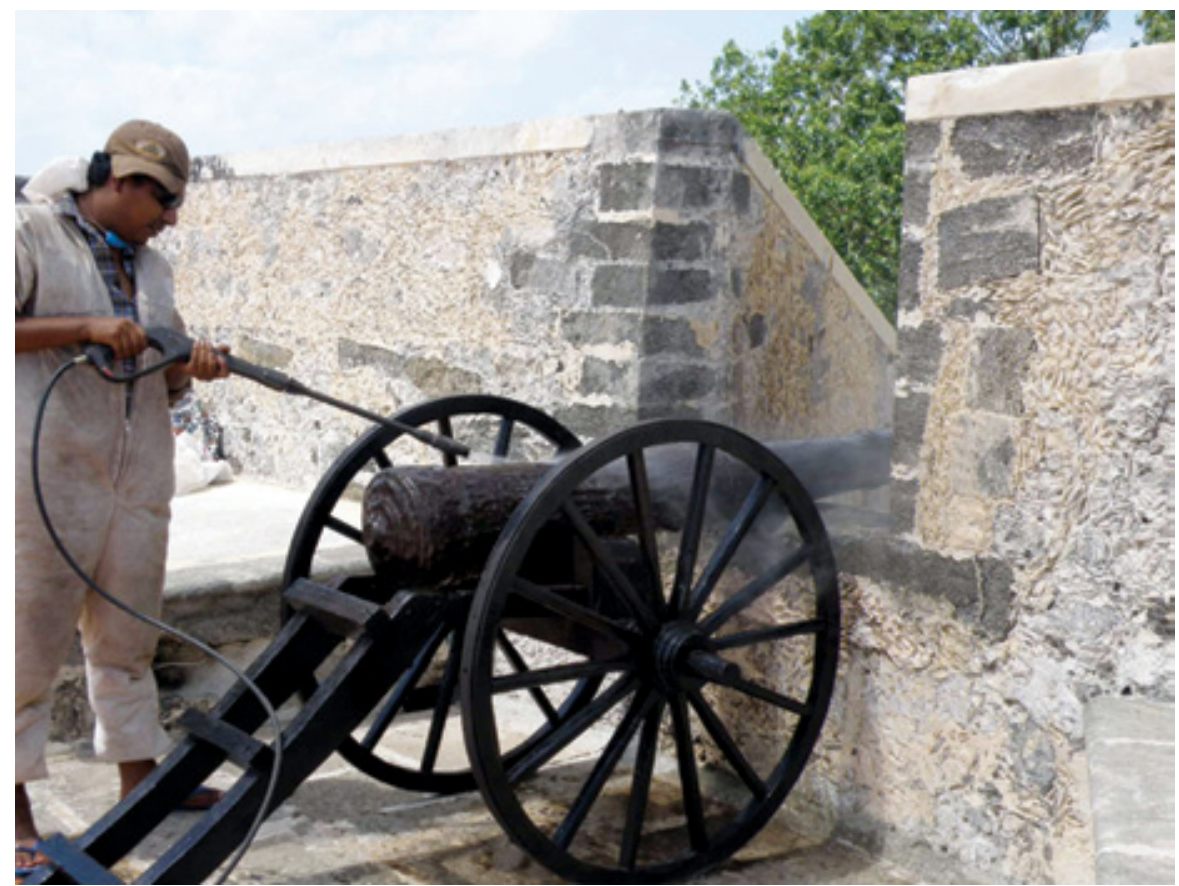

FIGURA 3. Lavado a presión con agua destilada en un cañón del Fuerte de San Miguel durante la primera etapa del proyecto "Restauración de vestigios de artillería" (Fuente: Arano Recio 2008; cortesía: Archivo Fotográfico de la Sección de Restauración del Centro INAH-Campeche).

cañón en un lapso de 10 semanas. Así, entre una y otra temporadas de campo se ha logrado estabilizar $64 \%$ de la colección de cañones de la ciudad de San Francisco de Campeche, y solamente queda por intervenir el porcentaje de piezas que el catálogo dictamina como en buen estado de conservación.

\section{Acciones de conservación}

La propuesta de conservación directa para el caso de los cañones de la ciudad de Campeche consistió en crear una capa de óxido artificial sobre el metal sano, que se trató con un estabilizador de herrumbre. Los procedimientos realizados se listan a continuación:

1. Limpieza y remoción de productos de corrosión por medios mecánicos. El hierro, al corroerse, se transforma en una capa poco densa y porosa, bastante más gruesa que el metal que le dio origen. Por su permeabilidad y agrietamiento, la costra de productos de corrosión, debajo de la cual se conserva la morfología de los cañones, capta el agua lluvia, la cual promueve los mecanismos de degradación. El objeto de este procedimiento de limpieza y remoción —que ha sido recomendado por Plenderleith (1967), Hamilton (1976), Pearson (1987) y Cronyn (1995) para objetos en hierro de procedencia marina cuya degradación es similar a la de los cañones que nos ocupan, expuestos a condiciones ambientales tropicales- es eliminar todos los productos de corrosión que no estén bien afianzados a la pieza, tratando de dejar solamente, perfectamente adherida y compacta, una delgada capa de ellos. Con este fin se utilizaron martillos, cinceles, lijas y máquinas con discos de lijas de carburo de silicio.

2. Lavado del cañón con agua destilada a presión. Para este proceso se utilizó una hidrolavadora conectada a un tambo de 250 I de agua destilada, que se transportaba en un vehículo oficial.

3. Eliminación de cloruros por reducción electrolítica. El tratamiento, consistente en conectar a una corriente eléctrica directa el cañón y una lámina de acero inoxidable como 
cátodo y ánodo, respectivamente, en el que se utilizó como electrolito una solución en agua destilada de hidróxido de sodio $2-5 \%$, se realizó con compresas de algodón impregnadas por el electrolito, colocándolas de manera intermedia entre los electrodos.

4. Formación de una capa de óxido. Para crear una delgada capa de óxido en la superficie de metal se aplicó una solución de ácido nítrico $1 \mathrm{~mol}$.

5. Estabilización de productos de corrosión. Se aplicó una solución de ácido tánico en metanol al 5\% p/p. Dicho ácido actúa como un estabilizador de herrumbre: convierte los óxidos de hierro en fosfatos y tanatos férricos, los cuales poseen mayor resistencia a la degradación (Arano Recio 2008) (Figura 4).

\section{Conclusiones}

Con base en sus dos temporadas, el proyecto "Restauración de vestigios de artillería" corroboró la eficacia del tratamiento propuesto y evaluado en el proyecto FOMIX CAMP-2005C01-026, mediante el cual se logró disminuir la velocidad de corrosión de la colección de cañones expuestos a condiciones de cielo abierto en la ciudad de Campeche, en un orden de magnitud (disminuye 10 veces su velocidad de corrosión).

Con la investigación (Arano Recio 2008) que antecedió al proyecto citado se confirmó el valor de la colaboración multidisciplinaria y del trabajo conjunto interinstitucional que exige la conservación del patrimonio cultural en nuestro país.

Contrario a lo que se ha pensado, los cañones deben estar expuestos al aire libre en sitios en los que la luz solar incida la mayor parte del día, ya que al calentarse el metal se elimina la posibilidad de condensación de humedad y, por lo tanto, que se active la reacción de corrosión. Guardarlos en espacios techados y cerrados resulta nocivo, pues a causa del clima de la región sufrirían de mayor cantidad de horas de conden-

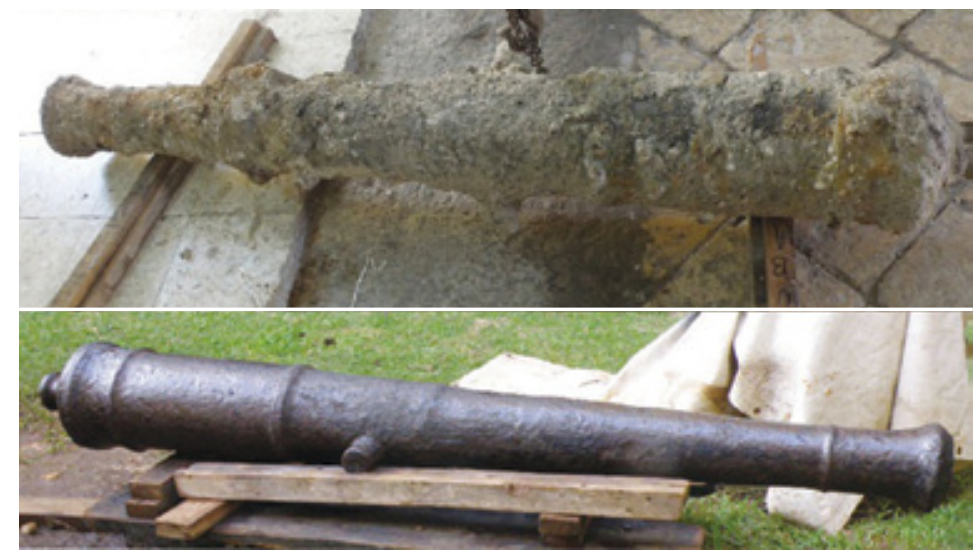

FIGURA 4. Cañón procedente de Ciudad del Carmen, Campeche, encontrado en el subsuelo durante la ejecución de obras públicas, restaurado por la Sección de Restauración del Centro INAH-Campeche en 2008. Se observa el cañón de hierro antes y después de su restauración (Fuente: Arano Recio 2008; cortesía: Archivo Fotográfico de la Sección de Restauración del Centro INAH-Campeche).

sación de humedad y, por ende, de corrosión.

Durante las temporadas 2009 y 2010 se ha logrado la estabilización de $64 \%$ de la colección de cañones. El personal que participó en el proyecto aprendió no sólo a realizar cabalmente este trabajo sino también a valorar la importancia de la permanencia de los vestigios de artillería en Campeche.

\section{Agradecimientos}

Al INAH (Centro INAH-Campeche); al Consejo Nacional de Ciencia y Tecnología (Conacyt), por la beca otorgada a la autora de este INFORME, a través de los proyectos FOMIX CAMP2006-01-23492 y 2005-C01-026, y al proyecto SEP-2004-C01-46434, Conacyt; a la Fundación Desarrollo Educacional de Campeche (Fundec); al Cicorr-UAC; al Departamento de Física Aplicada del Centro de Investigación y de Estudios Avanzados (Cinvestav)-Mérida, del Instituto Politécnico Nacional (IPN), en particular a la doctora Patricia Quintana, al maestro Daniel Aguilar y al doctor Pascual Bartolo Pérez.

Asimismo, al Instituto de Física de la Universidad Nacional Autónoma de México (IF-UNAM), en especial al doctor Jesús Arenas, y a los maestros Roberto Hernández y Diego Quintero.

\section{Referencias}

Arano Recio, Diana

2008 "Conservación de cañones de fundición de hierro del periodo colonial, que forman parte del patrimonio cultural de la ciudad de San Francisco de Campeche", tesis de Maestría en Ciencias Marinas, México, Facultad de Ingeniería/UAC.

Carrero Blanco, Luis

s. f. Arte naval militar. I. Las armas navales (Del bodoque a la bomba atómica), Madrid, Editorial Naval.

Carvajal Correa, Marco

2004 Inventario de bienes culturales que se encuentran bajo la jurisdicción del Centro INAH-Campeche, Campeche, Dirección de Museos/Centro INAH-Campeche.

Cronyn, Janet $M$.

1995 Elements of Archaeological Conservation, Nueva York, Routlegde.

Díaz Fuentes, Belinda

2010 "Arqueología de los objetos metálicos de la época colonial en Campeche. Catálogo de anclas, balas y cañones", tesis de Licenciatura en Arqueología, México, ENAH-INAH: 113-142.

Stefanescu, Doru M. y Roxana Ruxanda 2004 "Metallography: an introduction", Metallography and Microstructures, ASM Handbook, ASM International, 9: 3-15. 2004 "Solidification structures of steels and cast irons", Metallography and Microstructures, ASM Handbook, ASM International, 9: 97-106. 
Novegil-Anleo, F. J., J. J. Beltrán, J. Minotas, K. E. García, A. L. Morales, G. Pérez, J. Tobón y C. A. Barrero

2005 "Acción de los convertidores de herrumbre en óxidos de hierro", Revista de la Sociedad Colombiana de Física, 37: 203-206.

González Sánchez, Jorge

2005 "Proyecto Fondo Mixto Conacyt", convocatoria 2005, clave CAMP2005-C01-026, Campeche, Gobierno del Estado de Campeche.

Hamilton, Donny Leon

1976 Conservation of Metal Objects from Underwater Sites: a Study in Methods, Austin, The Texas Memorial Museum.
Kumar Sinha, Anir

2003 Physical Metallurgy Handbook,

Nueva York, McGraw-Hill.

Pearson, Colin 1987 Conservation of Marine Archaeological Objects, Londres, Butterworth.

Plenderleith, Harold J.

1967 La conservación de antigüedades y obras de arte, Madrid, Instituto Central de Conservación y Restauración de Obras de Arte, Arqueología y Etnología.

Radzikowska, Janina M. 2004 Metallography and Microstructures of Cast Iron, Metallography and Microstructures, ASM Handbook, ASM International, 9: 565-587.
Reyes Trujeque, Javier

1999 "Influencia de los principales factores climáticos y de la calidad del aire sobre la corrosión atmosférica de los metales en la costa sureste del Golfo de México", tesis de Maestría en Ingeniería Ambiental, Veracruz, UV.

Veleva, L.

2003 "Atmospheric corrosion", Metals Handbook Corrosion Fundamentals. Testing and Protection, (10 a ed.), ASM International, 13A.

\section{Resumen}

Por su relevancia histórica, los cañones manufacturados en hierro colado gris están expuestos en espacios públicos a cielo abierto en la ciudad de San Francisco de Campeche, México, donde predomina una atmósfera característica de clima cálido tropical húmedo, que es clasificada con una potencia corrosiva media- alta.

Durante la investigación se realizaron diferentes análisis a cañones de hierro gris con la finalidad de identificar la composición de los productos de corrosión. Las pruebas electroquímicas permitieron conocer el comportamiento del metal de los cañones ante el agua lluvia, además de evaluar la formación de pátinas estables usando $5 \%$ de $\mathrm{NaOH}$ y $5 \%$ de ácido tánico en solución.

Adicionalmente, se implementó una estrategia de conservación que puso en práctica un programa de mantenimiento y preservación a la colección de 107 cañones basada tanto en los resultados científicos obtenidos como en la colaboración interinstitucional entre la Universidad Autónoma de Campeche, el Gobierno del Estado de Campeche, la Secretaría de Desarrollo Social y el Instituto Nacional de Antropología e Historia (todas ellas instituciones de México).

\section{Palabras clave}

Conservación; artillería; electroquímica; hierro; trópico

\section{Abstract}

Due to their historical importance, the artillery cannons manufactured in grey cast iron are exhibited in public open places at the San Francisco de Campeche city, Mexico, where a hot, humid tropical climate prevails, which is classified as causing a medium to high level of corrosion.

During this research, several analyses were carried out to grey iron cannons in order to determine the chemical composition of their corrosive products. The electrochemical tests were used to understand the behaviour of the cannons' metal under the rain, and also to evaluate the formation of stable patinas using $5 \% \mathrm{NaOH}$ and a $5 \%$ tannic acid solution. Furthermore, a conservation strategy was implemented, that set in action a maintenance and a preservation program for a collection of 107 cannons, all which was based on scientific results derived from the above-mentioned research and on the inter-institutional collaboration of the Universidad Autónoma de Campeche (Autonomous University of Campeche), the government of the state of Campeche, la Secretaría de Desarrollo Social (Secretariat of Social Development), and el Instituto Nacional de Antropología e Historia (National Institute of Anthropology and History) (all of them institutions in Mexico).

\section{Key words}

Conservation; artillery; electrochemistry; iron; tropical

Título en ingles: Project: Restoration of Artillery Relics: Aspects regarding the Research and Conservation of Iron Alloy Cannons from the City of San Francisco de Campeche, Mexico 